\title{
Discussing diabetes, palliative and end of life care: choosing the 'right' language
}

\author{
Trisha Dunning ${ }^{1,2} \wedge$, Peter Martin ${ }^{3}$, Sharyn Milnes ${ }^{1,3,4}$, Nick Simpson ${ }^{3,4}$, Georgie B. Lee $^{1,3} \wedge$, Neil Orford ${ }^{3,4,5}$ \\ ${ }^{1}$ Centre for Quality and Patient Safety Research, Barwon Health Partnership, Deakin University, Geelong, Australia; ${ }^{2}$ School of Nursing and \\ Midwifery, Deakin University, Geelong, Australia; ${ }^{3}$ School of Medicine, Deakin University, Geelong, Australia; ${ }^{4}$ Intensive Care Unit, University \\ Hospital Geelong, Geelong, Australia; ${ }^{5}$ Australian and New Zealand Intensive Care Research Centre (ANZIC-RC), Department of Epidemiology \\ and Preventive Medicine (DEPM), Monash University, Clayton, Australia \\ Contributions: (I) Conception and design: T Dunning, P Martin; (II) Administrative support: S Milne, GB Lee; (III) Provision of study materials or \\ patients: T Dunning, N Orford; (IV) Collection and assembly of data: T Dunning, S Milne; (V) Data analysis and interpretation: T Dunning, N \\ Simpson; (VI) Manuscript writing: All authors; (VII) Final approval of manuscript: All authors. \\ Correspondence to: Professor Trisha Dunning, RN, CDE, MEd, PhD, AM. Chair in Nursing, Centre for Quality and Patient Safety Research, Barwon \\ Health Partnership, School of Nursing and Midwifery, Deakin University, KHERB-Building, C/- The Geelong Hospital, Ryrie Street PO Box 281, \\ Geelong Vic 3220, Australia. Email: trisha.dunning@deakin.edu.au.
}

Background: Palliative care and advance care planning are important components of diabetes and other chronic disease management plans. Most people with diabetes do not have conversations about palliative/end of life (EOL) care or advance care directives; often because diabetes clinicians are reluctant to discuss these issues. Guidelines for conversations and decision aids can assist shared decision-making for both clinicians and patients. The aim was to co-design information with older people with diabetes, families and clinicians to facilitate conversations about palliative and EOL care and to identify the language (words) people with diabetes, families and diabetes clinicians use to discuss death and dying

Methods: We co-designed and tailored the information with advisory groups: (I) older people with diabetes/families, (II) interdisciplinary expert clinicians and undertook a scoping literature review to identify relevant content. The penultimate versions were independently evaluated in focus groups and via written feedback from representative stakeholder clinicians, people with diabetes and international experts in communication, palliative care and diabetes.

Results: The information met design and language criteria: 62 people participated in focus groups (48 clinicians, 14 older people with diabetes and 4 family members). There were important differences between people with diabetes and diabetes clinicians concerning the words used to refer to death: 'the language of death'. Diabetes clinicians choose soft words/euphemisms such as 'passed away' and 'gone' and indicated they mirrored patient language. People with diabetes preferred clear language and indicated euphemisms were confusing and misleading. Diabetes clinicians, rarely encountered death in their practice and indicated they lacked education about how to discuss diabetes EOL care.

Conclusions: Co-design with key end-users improved relevance to these groups. Diabetes clinicians prefer to use euphemisms for death and would benefit from education and strategies to help them initiate conversations about EOL diabetes care.

Keywords: Diabetes; co-design; advance care planning; decision aids; communication

Submitted Aug 04, 2020. Accepted for publication Dec 17, 2020.

doi: 10.21037/apm-20-1548

View this article at: http://dx.doi.org/10.21037/apm-20-1548

^ ORCID: Trisha Dunning, 0000-0002-0284-1706; Georgie B. Lee, 0000-0002-4343-3020. 


\section{Introduction}

The Australian population is ageing, and Australia has one of the highest proportions of centenarians in the world. Older age is a risk factor for diabetes. The underlying pathophysiology of diabetes exacerbates the effect of ageing on metabolic processes and accelerates the progression of complications that affect life expectancy (1). Diabetes is the most prevalent, costly and complex chronic disease and is associated with deaths due to cardiovascular disease, cancer, stroke and renal disease $(2,3)$.

The International Diabetes Federation (IDF) indicated one person with diabetes in the world dies every six seconds (4). The World Health Organisation (WHO) suggested healthy ageing should be considered from a person's functional ability and focus on enabling them to do the things they value, as well as on preventative care and cure (2). Although intensive blood glucose control ('tight control') is important to prevent complications early in the course of diabetes, the risks often outweigh the benefits. Older age and the development of functional and cognitive impairments affect quality of life and reduce life expectancy (5). Initiating palliative care $(\mathrm{PC})$ with usual diabetes care could improve comfort, quality of life, and help people maintain personal dignity and autonomy and have a 'good death' (6).

Many people with diabetes live a long time with significant disease and require several medicines (polypharmacy) and lifelong self-care to manage their diabetes. They often develop significant cumulative disease and treatment and self-care burdens, which means they require complex care in all settings and could benefit from comprehensive geriatric and palliative care assessments and end of life (EOL) care planning (7) rather than focusing on 'tight blood glucose control,' which may be unrealistic and unsafe (8). Over $50 \%$ of Australians indicate they have at least one chronic condition and between $60-87 \%$ aged 65 and older have more than two chronic conditions (3).

People with diabetes tend to follow a similar disease trajectory; the chronic disease trajectory, to $\operatorname{EOL}(9,10)$. However, a person's journey within the trajectory and across trajectories and their life expectancy is highly individual. Some of the burden and associated suffering could be alleviated using a palliative approach with usual diabetes care $(6,11)$. Thus, experts recommend proactive screening for diabetes to ensure it is diagnosed early, adopting a life course approach to managing diabetes (12) and tailoring or personalising care, including medicines, HbAlc and blood glucose ranges, to achieve optimal outcomes.
Optimal outcomes include managing the presenting condition, recognising and managing deterioration and recognising when the person is dying $(1,4,5)$. These are compelling reasons to include EOL and palliative care (PC) into diabetes management guidelines and were the impetus for our EOL PC research program, including the current study.

The need to plan for old age and EOL and to accept death as inevitable and sometimes desirable is re-emerging in modern health care. For example, the WHO promotes dying as normal and suggested optimal EOL care affirms life (2).

Older age and death are normal, important aspects of people's life journeys. In addition, the Clinical Practice Guidelines for Quality Palliative Care (13) recommend service providers and clinicians integrate $\mathrm{PC}$ into the care plans of people with serious illnesses and stressed that $\mathrm{PC}$ is not restricted to PC specialists: it is the responsibility of all clinicians.

\section{Aims}

(I) Co-design and formatively evaluate tailored information to help older people with diabetes, their families and clinicians discuss palliative and EOL care.

(II) Summative evaluate the information with clinicians and people with diabetes/families who were not involved in co-designing the information and use their responses to revise the information if/where necessary

(III) Identify the language (words) diabetes clinicians, people with diabetes and families use to discuss death and dying.

(IV) Undertake independent, international expert review of the information using the Well Written Information for Consumers (14).

We present the following article in accordance with the MDAR checklist (available at http://dx.doi.org/10.21037/ apm-20-1548).

\section{Methods}

The study consisted of two inter-related phases to develop three versions of the information about ACP tailored with (I) older people with diabetes, (II) families and (III) An interdisciplinary group of clinicians and is reported in detail elsewhere (11). The information was developed collaboratively with three advisory groups (AGs) and a designer, who met separately to discuss the draft content 
and design and layout of the information. An iterative process was used where the initial draft information was developed by the research team, discussed with the AGs and revised until consensus was reached (15) and the AGs felt the information was ready to be evaluated by the broader community of key end-users represented by the three AGs.

The information was designed to be consistent with the Victorian Medical Treatment Planning and Decisions Act 2016 (Vic), Medical Treatment Planning and Decisions Act: Summary for Health Professionals 2018, and the Australian Commission of Quality and Safety in Health Care Standards (16).

\section{Summative evaluation}

The sampling population for the summative evaluation consisted of older people with diabetes and family carers living in Victoria and medical, nursing and other clinicians from health services and academic settings around Australia. Older people with diabetes and family members were recruited through Barwon Health diabetes services, Diabetes Victoria and researcher networks to encompass a range of socioeconomic groups and various health literacy levels. The sample included both males and females aged 65 years or older. Clinicians were recruited through professional associations such as the Australian Diabetes Educators Association (ADEA) and snowball sampling where clinicians recommended participation to colleagues.

The Well Written Information for Consumers (14) was used as a framework to guide the design and content of the ACP information. In addition, the international experts used these criteria to evaluate the penultimate versions of the information to assess the content, development process and the potential effectiveness of the information.

\section{Data collection process}

Data were collected in focus groups and from participants providing written feedback about the information via mail or email if they could not attend a focus group to enable broad consultation and ensure a variety of opinions were included. We aimed to recruit 30 participants per group (clinicians, people with diabetes and families) and to include $5-10$ participants in each focus group $(n=90)$ to enable meaningful discussion to occur and was likely to identify the full range of important opinions about the information (data saturation) $(15,17)$.

The interviewer (TD) followed standard focus group procedures (18). A topic guide based on Currie et al. was used to guide the discussion (14). The focus group discussion was audiotaped, and field notes were recorded to capture non-verbal information and supplement the audiotaped information where the conversation was indistinct. These data were translated verbatim for analysis.

\section{Independent expert review}

Four people, not involved in the AGs or the research team (TD, PM, SM, NO, GL, NS), assessed the information using Currie et al.'s Criteria (14). They were experts in communication, geriatrics, diabetes and palliative and EOL care and were recruited through the researchers' networks. They independently reviewed each version of the information at the document level, considered relevance to the proposed target audiences in their assessment, and returned their assessments anonymously.

\section{Data analysis procedures}

Focus group data were analysed using the Framework Method (19) to identify emerging themes/issues. The framework method comprises five stages: becoming familiar with the data; identifying a thematic framework; applying the index or thematic framework systematically to the data; rearranging the data according to the part of the thematic framework they relate to; and mapping and interpreting the data, which enabled isolated research findings to be interpreted and appropriate themes to be identified (20). As indicated, the researcher's (TD) final interpretation of the data was verified with the three AGs (member checking). The four independent reviews were analysed and the data combined to arrive at a consensus assessment.

\section{Ethical issues}

Ethical issues concerned voluntary informed consent to participate and the possibility of emotional distress discussing an emotive topic. All potential participants and members of the AGs and expert reviewers were informed about the emotive nature of the research before they agreed to participate. Lifeline and Beyond Blue counseling services contact details were included in the patient information and consent forms. Written informed consent was obtained from all participants in the study. The study was conducted in accordance with the Declaration of Helsinki (as revised in 2013). Approval to conduct the study was obtained 
from both the Barwon Health Human Research Ethics Committee [09/120] and the Deakin University Human Research Ethics Committee [2017-258].

\section{Results}

Sixty-two people participated: 48 clinicians (doctors, nurses, pharmacists) 14 older people with diabetes, including both males and females, all aged 65 years or older, and four family members, while this was 28 fewer than the target sample size of 90 , it achieved a response rate of $70 \%$. All participants indicated the content was appropriate and easy to understand; however, some clinicians were concerned about the words dead, dying and died, which they described as 'the language of death.' Most people with diabetes felt these terms were clear and unambiguous. The older people with diabetes AG specifically asked that these words be used when the information was developed. Thus, the 'language of death' emerged as the dominant theme and had five inter-related sub-themes:

(I) Clinical area and practice settings.

(II) Need for clear language to enhance meaning.

(III) Importance of 'mirroring patient language.'

(IV) 'Hiding' death.

(V) Organizational and social culture and policies.

\section{Language of death}

Most clinicians indicated they did not use the words 'dying' and 'died/dead' when conversing with patients because they felt uncomfortable using these words, were 'scared' of the terms and preferred to use 'softer' words (death euphemisms) such as 'passed away' and 'gone' to describe death. Reasons for using death euphemisms included reducing their own and people with diabetes' discomfort. For the purposes of this paper, 'euphemism' refers to mild or innocuous words or expressions used in place of words that could be offensive or unpleasant.

Clinicians reported they used 'gentler' more sensitive and respectful words during difficult times for patients and families.

We try to soften it - the language around death (Diabetes Clinician, FG3).

'Passed away' is more pleasant sounding, not as harsh or abrupt (written feedback) (Diabetes Clinician FG2).

Many clinicians, especially diabetes clinicians described the terms 'dying' and 'death/died' as harsh because they suggest finality. They indicated telling a patient they were dying or family their family member had died was 'final', upsetting and distressing, and contributed to clinicians' reluctance to use those words.

It [died] is very strong and final wording (Clinician, FG3).

\section{Clinical area and practice setting}

The practice setting also influenced clinicians' word choice. Clinicians and people with diabetes/families suggested the level of comfort about openly discussing death varied amongst clinicians, their work setting and level of professional experience with death. For example, working in a setting that frequently requires clinicians to care for dying people could mean these clinicians are more comfortable using the words 'dying' and 'died/dead' because it is part of their usual language. Whereas clinicians working in areas such as diabetes care where they do not frequently use these terms or 'confront death' can result in these clinicians feeling less comfortable discussing death, consequently they use softer words.

One clinician said:

It depends on where you work. If you don't deliver that message very often you probably wouldn't find it easy. But if you work in an area where you're delivering that message a bit too much, or on a frequent basis, you probably would find it easier to use the language 'died'. You would be more de-sensitised to it. (FG2).

Clinicians also said they found it more difficult to use the words 'dying' and 'died/dead' with patients receiving disease-modifying treatment where the intention was curative, even when the person had a life-limiting illness. Although both type 1 and type 2 diabetes reduce life expectancy compared to the general population it is not usually regarded as a life limiting illness. Diabetes clinicians said these terms were inappropriate to use with patients who were 'not dying and could be detrimental to or diminish hope for them.'

However, if the person was receiving PC or EOL care, clinicians said it was easier and more appropriate to use the words 'dying' and 'died/dead.'

I think a PCU [palliative care unit] environment is different to an acute care setting or in outpatient settings. The view in acute is that you will be saved and recover and go home, whereas in PCU people are very much more comfortable about death (FG3).

\section{Need for clear messages}

Conversely, older people with diabetes and family members indicated they preferred clear and unambiguous language to 
describe and explain death to avoid misunderstanding. They suggested clinicians' unwillingness to have frank, open conversations about dying hindered the open discussions older people with diabetes and family members wanted to have and resulted in lost opportunities for clinicians to inform and educate people about a significant life event. older people indicated clinicians' reluctance to openly discuss deterioration towards death also created a barrier to connection and care. Further, older people with diabetes indicated being able to talk about their EOL was an integral aspect of their care, especially when they had diabetes complications.

Listening emerged as an important aspect of PC/EOL conversations. One man said he said he wanted clinicians to 'be present and listen to my concerns:'

I feel it is important for staff to be caring enough to ask if there is anything you [the patient] needs to talk about [EOL].... to listen, hear and respond (Person with diabetes, written feedback).

This comment could reflect the tension between clarity and respect, which are not mutually exclusive, although clinicians find it challenging to do both simultaneously, which can compromise clarity.

One family member discussed how she wanted clear messages when her relative was dying:

But at that stage do you want it [the language] to be that sensitive? ..t both my parents have been through palliative care. So you just want to hear 'this is the situation. They're dying.' So I would prefer them [clinicians] to say 'they're dying' (family member FG4).

Clinicians also discussed the importance of using clear language to communicate about death, which was contradictory to their long discussions about using 'softer' euphemisms and avoiding using 'harsh' words such as death. Further, they shared stories about how replacing the word 'dead' with a euphemism resulted in confusion and misunderstanding among clinicians and family members. A nurse said:

Not using the direct terminology [clear words] is a big issue. I reflect on a time when I was in a family meeting and the consultant was telling the family that their loved one had died and said they've 'gone' After the consultant left the family said 'where bas be [the patient] gone?' (FG1).

Another nurse responded:

There's been a number of times I've had where somebody's said 'we've lost so-and-so.' and some of the funny stories I've heard It can be really confusing. And then it is hard conversation to bring it back to tell them that the person died (FG2).
Clinicians indicated inadequate interprofessional communication and documentation also caused confusion. An intern said:

I did a project in a hospital about how the decision not to resuscitate was communicated among clinicians and family members. And it wasn't working among clinicians. They wouldn't say 'do not resuscitate'. They said 'not for 666' and that's the telephone code [resus code].

Another doctor stated:

I still see written in the medical notes 'patient passed away at 0600' and I feel a little disappointed... I think when someone is dying or dies the clinicians need to gently say that (clinician written feedback).

\section{Mirroring patient's language}

Clinicians also discussed the importance of 'mirroring' the language their patients and family members used to enhance communication and understanding. 'Mirroring' patients' language requires active listening and could mean clinicians might/might not use the words 'dying' and 'died/ dead' to show the individual and family they are hearing and respecting their preferred terms for death. Other reasons for using the same language as their patients and family members were to accommodate their patient's health literacy level and to meet 'in the middle.'

That is, clinicians adopted the words their patients used to connect with them and to foster a therapeutic relationship. For example:

It's part of matching and mirroring too. If a family member uses the word 'died', then you're going to use that term too. You're going to use the language the family useIt's part of the therapeutic relationship you bave with the family, using the terminology that the family use (Clinician FG3).

Further, clinicians equated using the language the family used as a respectful way to avoid words that could cause offense.

You need to try and find some middle ground and be less offensive. In many ways you have to try to be respectful and I think the word 'passed on' is not about being disrespectful, but about being as respectful as possible in a time that's going to be very difficult for the family (Clinician FG3).

Other clinicians stressed that it was important for them to have a clear understanding of what the language meant when mirroring patient/family language to avoid misinterpreting words and miscommunication, one said:

I think it's okay to reflect someone's language when you're talking about [death].. As long as you both [family member 
and clinician] know what you're talking about and that's clear (Clinician FG1).

Interestingly, people with diabetes also 'mirror' clinicians' diabetes language' (dialect) to enhance communication, even when they do not like specific words they regard as negative and labeling such as diabetic, victim and sufferer (6). Thus, mirroring language is a two-way process that can enhance or confound clear communication.

\section{Hiding death to protect other people}

Some clinicians said they did not use 'died/dead' to protect other patients in the area from distress. For example, if a patient died on a ward, clinicians did not want other patients to hear the patient had died so they used 'code' words to hide the death. The rationale for hiding death in this context was similar to reasons previously discussed, in that they did not want other patients to be distressed, upset or scared by the occurrence of a death. For example:

I think that raises the issue too, if you're working in a hospital environment, you don't really want to say 'Mrs Smith s died' but if you said 'Mrs Smith 555' [example of a code word for death] it's getting away from the reality and protecting other people around who might be upset that somebody had passed away (Clinician FG3).

Some clinicians and family compared current settings where people are usually hospitalised during the dying process to death in 'the olden days' where people died at home surrounded by family and often friends and where people were more familiar with death. A doctor said:

Death is so bidden now. I think a couple of generations ago death was normalised. And it happened at home and the family were all around and these days patients end up in hospital wards with the curtains and the doors closed and it's an optional thing whether they see it. It's not the norm (Clinician FG1).

Family members indicated dying in hospital created a barrier to family being completely involved in or present during the dying process, which removed them from the death. They felt medical technology and interventions such as ventilators, intravenous lines, infusions, cardiac monitors and drain tubes contributed to the sense that death is hidden.

For a long time we have been shielded from death, with most people dying in hospital, sometimes surrounded with technology (Family member written feedback).

\section{Organisational and social culture}

Some individuals in all participant groups (people with diabetes, family members and clinicians) indicated they were reluctant to use the words 'dying' and 'died/dead,' for cultural reasons.

I think because we're removed so much from it [death] in our current way of life. Some people don't see it as much and death is whisked away from people (Family member FG3).

Clinicians indicated they are conditioned to use soft language because they hear it from other clinicians. Thus, euphemisms have become a cultural expectation within healthcare settings. The media also use euphemisms, for example:

Most clinicians are socialised to say 'passed away'...I think it's the way society s socialized it, isn't it? In the media nobody really talks about 'death.' (Clinician FG2).

\section{Discussion}

The current study is a key part of our diabetes PC and EOL research program that encompasses communication (21-23). The research includes interviews with older people with diabetes receiving $\mathrm{PC}$ at their EOL, their families and clinicians. These interviews and other research revealed that people with diabetes receive insufficient information to help them understand their personal health risks and how to manage those risks $(11,21,24)$ and highlighted that all parties need information about how to discuss and plan for EOL care. This is a well know finding in the palliative care literature but is a relatively new finding with respect to diabetes clinicians

Our previous research showed people with diabetes are willing and want to discuss EOL care in the last 12 months of life and to use the words death and dying (25), which is consistent with other research. However, diabetes clinicians are often reluctant to initiate conversations about these key life transitions (26), consequently costly, burdensome and futile care often continues unnecessarily (27). In addition, diabetes is not generally included on information about life limiting illnesses, although it is known to reduce life expectancy (28). It is difficult to predict prognosis, although some general indicators of declining life expectancy such as heart disease, COPD, stroke, renal disease, motor neurone disease, Parkinson's disease, multiple sclerosis and dementia can be helpful starting points (28). Prognosis refers to the way an individual's illness and overall health is likely to evolve.

Mirroring language emerged as a relatively new concept with respect to diabetes in our study. As indicated, mirroring can be an aspect of respect and listening. It can act as a 
Table 1 Process considerations for initiating conversations about palliative and end of life care

Use a values-based approach rather than focusing on medical decisions

Conversations can be planned or opportunistic

If planned ask the individual who they would like to be present during the conversation and have all relevant information and documents ready

Ensure the environment is confidential and welcoming

Ensure the person brings any communication aids they need with them, e.g., spectacles and hearing aids

Present the information in easy to understand words and formats and assess their understanding

Frame the conversation as part of the individual's life story. Use own knowledge of life expectancy in similar conditions

Recognize and respond to verbal, non-verbal and emotional cues appropriately

Table 2 Some strategies for initiating conversations about palliative and end of life care

Some useful questions that can help start a conversation include the following: these questions should not be used as a 'tick box' list. They must be personalized to the individual and the situation. The questions need not be asked in any particular order

- What do I need to know about you to help me give you the best possible care and advice?

- What things make your life worth living or matter to you? Rather than asking about quality of life.

- What does suffering mean to you?

- What does a good death mean to you?

Conversations involve two or more people who speak, take turns to speak, interrupt and listen.

bridge to one's own experiences and enable conversational flow. Interestingly, patients also use mirroring to aid communication. For example, people with diabetes learn to speak the 'diabetes dialect' and use terms such as HbA1c, diabetic and glucose-lowering medicine names, which is a form of mirroring (29).

Mirroring may also contain elements of health literacy. Health literacy is important to quality care and can affect patients' decisions and actions (30). However, clinician health literacy is rarely considered and can also have a profound effect on outcomes. Our study suggests the clinician participants had low levels of literacy concerning PC and EOL care, which could compromise communication. Significantly, discussing EOL is highly emotive and people can take longer to understand and process information, which is one reason it is important to start a conversation and give people time to process the information and its implications. Documenting the things that give meaning and purpose to ones' life (values) may be a good starting point because it is less emotive, and values rarely change over a lifetime where care preferences can and do change.

Two other significant findings concerning language emerged from the study: Diabetes Clinicians, like many clinicians in other specialties preferred to use euphemisms to avoid using the language of death when discussing PC and EOL, and diabetes Clinicians are not educated to discuss declining life expectancy or to begin ACP. In contrast, people with diabetes and family members were more comfortable using clear language and terms such as dead and died. These issues emerged in our other studies and highlight to need to incorporate such training in all clinician under and postgraduate programs. Significantly, our research suggests enhancing clinicians' communication skills for EOL conversations may improve patient outcomes (22).

The literature review conducted for the study identified 34 barriers to optimal communication most of which emerged in our study (31). An organisational focus on cure and prevention encourages the use of futile interventions (32). Sadly, ACDs are often documented at the end of a Medical Emergency Team call (33), which may lead to inaccuracies and increased stress.

Diabetes Clinicians indicated they find it difficult to talk about death in an environment that focuses on prevention and cure, and their discomfort is increased when they have inadequate communication skills and training, inadequate knowledge about the various cultural aspects surrounding death and dying and prognostic challenges. It is imperative that strategies are adopted to address these barriers, given that discussing EOL care is everybody's responsibility, not only the preserve of PC experts (34,35). Tables 1 and 2 outline some strategies clinicians can use to initiate conversations about $\mathrm{PC}$ and EOL. Table 3 provides guides for clinicians references legal and practical resources for end-of-life conversations with patients.

Knowing when and how to interrupt is a key skill. Older people need time to process the question in order to respond-a complex cognitive process, especially when 
Table 3 Web Links to the Medical Treatment Planning and Decisions Act and to our Suite of Information and Guidelines for initiating conversations with people with diabetes about advance care planning

The Victorian Medical Treatment Planning and Decisions Act (2016) became law on 12 March 2018. The suite of information draws on and is consistent with that legislation. The information will help clinicians, older people with diabetes and families understand The Act, when and how to start discussing these issues with older people with diabetes and how to develop an Advanced Care Directive https://www2.health.vic.gov.au/ hospitals-and-health-services/patient-care/end-of-life-care/ advance-care-planning/medical-treatment-planning-anddecisions-act

The suite of information developed as part of this study is available online on relevant websites including:

- Caresearch - A palliative care knowledge network funded by the Australian Government Department of Health (see https://www.caresearch.com.au/Caresearch/Default.aspx)

- The Australian Disease Management Association (see: https://adma.org.au/tools-and-resources/)

the topic is emotive. Interrupting can cause confusion and change the discussion and important issues might not be identified. Understand and accept that not everybody is capable of making informed decisions during a crisis and some people prefer certainty, i.e., to be 'told what to do.'

Prognostication is challenging. However, diabetes is known to reduce life expectancy compared to the general population and there are some well-described general indicators of declining life expectancy (30). In addition, our literature review for the current study revealed seven main diabetes-related factors associated with reduced life expectancy: duration of diabetes, glycaemia, comorbid load/ geriatric syndromes, severe hypoglycaemia, lower limb and foot disease, polypharmacy and comorbid depression (11).

Any or all of these conditions could indicate the need to start a conversation about PC/EOL and document an Advance Care Directive, given that many people with diabetes have other complications and co-morbidities that also reduce life expectancy: every concomitant chronic disease reduces life expectancy by 1.8 years (36). Thus, many aspects of comprehensive geriatric assessment are relevant to EOL care planning and identifying the actual and potential factors that compromise diagnosis and treatment (37). Documenting Advance Care Directive and clearly communicating values on which to base care decisions are important components of quality care (16), including diabetes care.

\section{Limitations of the study}

The findings might reflect a western perspective and language concerning death, which may differ from nonWestern cultures. The sample was relatively small, thus the findings might not apply to other countries or other diabetes clinicians, people with diabetes and family members within or outside Australia. However, the sample size was offset by sample heterogeneity, member checking and data saturation, which are strengths of the study. Codesign was also a significant strength of the study.

\section{Conclusions}

We co-designed EOL/PC information with older people with diabetes, families and clinicians to facilitate conversations about PC and EOL care (38-40). The purpose was to develop Guidelines for deciding palliative and EOL care with people with diabetes, as well as information booklets for older people with diabetes, health professionals and families caring for older relatives with diabetes (41).

Co-design with key end-users improved the relevance of the information to these groups. It enabled us to develop the content and formulate questions used in the evaluation, which enhanced understanding and catered to a range of literacy levels.

Diabetes Clinicians prefer to use euphemisms for death and would benefit from education and other strategies to help them initiate conversations about EOL as part of diabetes care.

Diabetes clinicians were reluctant to discuss life expectance, advance care planning and death. A discrepancy between the language diabetes clinicians preferred and the language older people with diabetes and families wanted, emerged .

The clinical relevance of the Guidelines is enhanced through information encompassing guidance in the Australian Commission on Safety and Quality in Health Care; National Safety and Quality Health Service Standards second edition (16) and the National Statement on Health Literacy (42). Significantly, the study helps explain some reasons why clinicians use death euphemisms.

\section{Acknowledgments}

The authors acknowledge the clinicians and people with 
diabetes and families who worked on the advisory groups and helped co-designing the information as well as those who participated in the summative and expert external evaluation.

Funding: This work was supported by a grant provided by the Diabetes Australia Research Trust [Y17G-DUNT].

\section{Footnote}

Reporting Checklist: The authors have completed the MDAR checklist. Available at http://dx.doi.org/10.21037/apm-201548

Data Sharing Statement: Available at http://dx.doi. org/10.21037/apm-20-1548

Peer Review File: Available at http://dx.doi.org/10.21037/ apm-20-1548

Conflicts of Interest: All authors have completed the ICMJE uniform disclosure form (available at http://dx.doi. org/10.21037/apm-20-1548). Dr. TD serves as an unpaid editorial board member of Annals of Palliative Medicine from Dec 2018 to Nov 2020, and reports grants from Diabetes Australia Research Trust, during the conduct of the study. The other authors have no conflicts of interest to declare.

Ethical Statement: The authors are accountable for all aspects of the work in ensuring questions relating to accuracy or integrity of any part of the work are appropriately investigated and resolved. Written informed consent was obtained from all participants in the study. The study was conducted in accordance to the Declaration of Helsinki (as revised in 2013). Approval to conduct the study was obtained from both the Barwon Health Human Research Ethics Committee [09/120] and the Deakin University Human Research Ethics Committee [2017-258]

Open Access Statement: This is an Open Access article distributed in accordance with the Creative Commons Attribution-NonCommercial-NoDerivs 4.0 International License (CC BY-NC-ND 4.0), which permits the noncommercial replication and distribution of the article with the strict proviso that no changes or edits are made and the original work is properly cited (including links to both the formal publication through the relevant DOI and the license). See: https://creativecommons.org/licenses/by-nc-nd/4.0/.

\section{References}

1. LeRoith D, Biessels GJ, Braithwaite SS, et al. Treatment of diabetes in older adults: an endocrine society clinical practice guideline. J Clin Endocrinol Metab 2019;104:1520-74.

2. World Health Organization. Strengthening of palliative care as a component of integrated treatment throughout the life course. J Pain Palliat Care Pharmacother 2014;28:130-4.

3. Australian Institute of Health and Welfare. Deaths among people with diabetes in Australia 2009-2014. Canberra, 2017.

4. Cho NH, Shaw J, Karuranga S, et al. IDF Diabetes Atlas: Global estimates of diabetes prevalence for 2017 and projections for 2045. Diabetes Res Clin Pract 2018;138:271-81.

5. Inzucchi SE, Bergenstal RM, Buse JB, et al. Management of hyperglycemia in type 2 diabetes: a patient-centered approach: position statement of the American Diabetes Association (ADA) and the European Association for the Study of Diabetes (EASD). Diabetes Care 2012;35:136479. Erratum in: Diabetes Care 2013;36:490.

6. Dunning T, Martin P. Palliative and end of life care of people with diabetes: Issues, challenges and strategies. Diabetes Res Clin Pract 2018;143:454-63.

7. McKechnie D, Pryor J, Fisher MJ, et al. Examination of the dependency and complexity of patients admitted to in-patient rehabilitation in Australia. Aust Health Rev 2020;44:143-52.

8. Kalra S, Sharma SK. Diabetes in the Elderly. Diabetes Therapy 2018;9:493-500.

9. Lynn J, Adamson DM. Living well at the end of life. Adapting health care to serious chronic illness in old age. Rand Corp Santa Monica, 2003.

10. Dunning P. The common disease trajectories: are they relevant to guide care as older people with diabetes progress towards their end of life? SM Gerontol Geriatr Res 2018;2:1011.

11. Dunning T MP, Orford N, Orellana L. Improving Care of Older people with diabetes, family members and clinicians communication and decisions about palliative and end of life care final report. Geelong Barwon Health Partnership Centre for Quality and Patient Safety Research Deakin University, 2019.

12. Lynch J, Smith GD. A life course approach to chronic disease epidemiology. Annu Rev Public Health 2005;26:1-35. 
13. Ferrell BR, Twaddle ML, Melnick A, et al. National Consensus Project Clinical Practice Guidelines for Quality Palliative Care Guidelines, 4th Edition. J Palliat Med 2018;21:1684-9.

14. Currie K, Spink J, Rajendran M. Well-written health information: a guide. Melbourne: Department of Human Services, 2000.

15. Jayasekara RS. Focus groups in nursing research: methodological perspectives. Nurs Outlook 2012;60:411-6.

16. Australian Commission on Safety and Quailty in Health Care. National Safety and Quality Health Service Standards. Sydney: ACSQHC, 2017.

17. Guest G, Namey E, McKenna K. How many focus groups are enough? Building an evidence base for nonprobability sample sizes. Field Methods 2017;29:3-22.

18. University of Kansas. Community Tool Box - Conducting Focus Groups 2018. Available online: https://ctb.ku.edu/ en/table-of-contents/assessment/assessing...focus-groups/ main.

19. Ritchie J, Spencer L. Qualitative data analysis for applied policy research. Analyzing qualitative data: Routledge, 2002:187-208.

20. Booth A, Carroll C. How to build up the actionable knowledge base: the role of 'best fit' framework synthesis for studies of improvement in healthcare. BMJ Qual Saf 2015;24:700-8.

21. Dunning T MP, Savage S, Duggan N. Guidelines for managing diabetes at the end of life 2010. Available online: http://www.adma.org.au/clearinghouse/doc_details/35guidelines-for-managing-diabetes-at-the-end-of-life.html

22. Orford NR, Milnes S, Simpson N, et al. Effect of communication skills training on outcomes in critically ill patients with life-limiting illness referred for intensive care management: a before-and-after study. BMJ Support Palliat Care 2019;9:e21.

23. Orford NR, Milnes SL, Lambert N, et al. Prevalence, goals of care and long-term outcomes of patients with life-limiting illness referred to a tertiary ICU. Crit Care Resusc 2016;18:181-8.

24. Roach P, Marrero D. A critical dialogue: communicating with type 2 diabetes patients about cardiovascular risk. Vasc Health Risk Manag 2005;1:301.

25. Savage S, Duggan N, Dunning T, et al. The experiences and care preferences of people with diabetes at the end of life: a qualitative study. J Hosp Palliat Nurs 2012;14:293-302.

26. Claessen SJJ, Francke AL, Engels Y, et al. How do GPs identify a need for palliative care in their patients? An interview study. BMC Fam Pract 2013;14:42-8.

27. Swerissen H, Duckett S, Farmer J. Dying well. Grattan Institute, 2014.

28. Thomas K, Armstrong Wilson J, et al. The Gold Standards Framework proactive identification guidance (PIG). Royal College of General Practitioners, 2016.

29. Dunning T, Speight J, Bennett C. Language, the "Diabetes Restricted Code/Dialect," and What It Means for People With Diabetes and Clinicians. Diabetes Educ 2017;43:18-26.

30. Burks AC, Keim-Malpass J. Health literacy and informed consent for clinical trials: a systematic review and implications for nurses. Nursing: Research and Reviews 2019;9:31.

31. Aslakson RA, Wyskiel R, Thornton I, et al. NursePerceived Barriers to Effective Communication Regarding Prognosis and Optimal End-of-Life Care for Surgical ICU Patients: A Qualitative Exploration. J Palliat Med 2012;15:910-5.

32. Murray SA, Kendall M, Mitchell G, et al. Palliative care from diagnosis to death. BMJ 2017;356: j878.

33. Jäderling G, Bell M, Martling CR, et al. Limitations of medical treatment among patients attended by the rapid response team. Acta Anaesthesiol Scand 2013;57:1268-74.

34. Nelson R. New Palliative Care Guidelines Demand 'Seismic Shift' in Care 2018. Available online: https://www. medscape.com/viewarticle/904367_print.

35. Palliative Care Australia. Standards for providing quality palliative care for all Australians: Palliative Care, 2005.

36. DuGoff EH, Bandeen-Roche K, Anderson GF.

Relationship between continuity of care and adverse outcomes varies by number of chronic conditions among older adults with diabetes. J Comorb 2016;6:65-72.

37. Overcash J, Ford N, Kress E, et al. Comprehensive Geriatric Assessment as a Versatile Tool to Enhance the Care of the Older Person Diagnosed with Cancer. Geriatrics (Basel) 2019;4:39.

38. Dunning T, Martin P, Orellana L, et al. Palliative and end of life care: information for older people with diabetes, 2018. Available online: https://adma.org.au/download/ palliative-and-end-of-life-care-information-for-olderpeople-with-diabetes/

39. Dunning T, Martin P, Orellana L, et al. Palliative and end of life care: information for families caring for older relatives with diabetes, 2018. Available online: https:// adma.org.au/download/palliative-and-end-of-life-careinformation-for-families-caring-for-older-relatives-with- 
diabetes/

40. Dunning T, Martin P, Orellana L, et al. Planning palliative and end of life care with older people with diabetes: information for health professionals, 2018. Available online: https://adma.org.au/download/revised-guidelinesfor-deciding-palliative-and-end-of-life-care-with-peoplewith-diabetes/

41. Dunning T MP, Orellana L, Orford N, et al. Guidelines for deciding Palliative and End of Life Care with People with Diabetes, 2018. Available online: https://adma.org.au/ download/revised-guidelines-for-deciding-palliative-andend-of-life-care-with-people-with-diabetes/

42. Australian Commission on Safety and Quailty in Health Care. National Statement on Health Literacy - Taking action to improve safety and quality. Sydney: ACSQHC, 2014.

Cite this article as: Dunning T, Martin P, Milnes S, Simpson N, Lee GB, Orford N. Discussing diabetes, palliative and end of life care: choosing the 'right' language. Ann Palliat Med 2021;10(4):3739-3749. doi: 10.21037/apm-20-1548 